\title{
Converging Multidimensional Sensor and Machine Learning Towards High-Throughput and Biorecognition Element-Free Multidetermination of Extracellular Vesicle Biomarkers
}

Caroline Y. N. Nicoliche, ${ }^{+, \neq \#}$ Ricardo A. G. de Oliveira, ${ }^{+, \#}$ Giulia S. da Silva, ${ }^{+, \neq \#}$ Larissa F. Ferreira, ${ }^{+, \neq}$Ian L. Rodrigues, ${ }^{\dagger}$ Ronaldo C. Faria, ${ }^{\S}$ Adalberto Fazzio, ${ }^{+, \epsilon}$ Emanuel Carrilho," Letícia G. de Pontes, "l Gabriel R. Schleder, ${ }^{+, \epsilon}$ and Renato S. Lima ${ }^{\star^{+,}}$

†Brazilian Nanotechnology National Laboratory, Brazilian Center for Research in Energy and Materials, Campinas, São Paulo 13083-970, Brazil. *Institute of Chemistry, State University of Campinas, Campinas, São Paulo 13083-970, Brazil.

SDepartment of Chemistry, Federal University of São Carlos, São Carlos, São Paulo 13565-905, Brazil.

${ }^{\epsilon}$ Center for Natural and Human Sciences, Federal University of ABC, Santo André, São Paulo o9210-580, Brazil.

IISão Carlos Institute of Chemistry, University of São Paulo, São Carlos, São Paulo 13566-590, Brazil.

\#These authors contributed equally to this work.

*Corresponding author: Renato Sousa Lima, e-mail: renato.lima@Innano.cnpem.br.

This supporting information contemplates information on:

Chemicals and samples
2. Device fabrication
3. Electrodes
4. Impedance analyses
5. Extracellular vesicle biomarkers
Data treatment
Data chemical diversification by the core
Electrode characterization
10. Classification of the mouse samples
1. Machine learning data to vesicle-containing mouse samples
2. Machine learning data to heavy metal-spiked lake samples
3. Specific determination of biomarker
14. Video caption




\section{Chemicals and samples}

Bovine serum albumin (BSA), 1-(3-(dimethylamino) propyl)-3-ethylcarbodiimide (EDC), 2-(N-morpholino) ethanesulfonic acid (MES), and uranyl acetate were purchased from Sigma-Aldrich (St Louis, MO). Sylgard 184 silicone and AZR 50XT resist were purchased from Dow Corning (Midland, MI) and Microchemicals (Ulm, Germany), respectively, whereas the magnetic beads (MBs, Dynabeads MyOne Carboxylic Acid) were acquired from Thermo Fisher (Waltham, USA). Monoclonal antibody (anti-p16) and p16 peptide were purchased from Imuny Biotechnology (Campinas, Sao Paulo). Distilled water was obtained with resistivity of $0.48 \mathrm{M} \Omega$ $\mathrm{cm}$ (Tecnal TE-2755, Piracicaba, Brazil). Lake samples were collected in the city of Campinas, Brazil. Calcium chloride $\left(\mathrm{CaCl}_{2} .2 \mathrm{H}_{2} \mathrm{O}\right)$, sodium chloride $(\mathrm{NaCl})$, and aluminum nitrate nonahydrate $\left(\mathrm{Al}\left(\mathrm{NO}_{3}\right)_{3} .9 \mathrm{H}_{2} \mathrm{O}\right)$ were provided by Synth (Diadema, Brazil). Phosphatebuffered saline (PBS) solution was purchased from Izon Science (Oxford, UK). Swiss mice were supplied by the Central Vivarium of the Faculty of Pharmaceutical Sciences (São Paulo State University). The mice were kept in polycarbonate cages with water and feed (Purina) in an air-conditioned place $\left(23 \pm 2{ }^{\circ} \mathrm{C}\right.$ and $56 \% \pm 2 \%$ relative air humidity) with photoperiod control every $12 \mathrm{~h}$. All procedures with animals were conducted according to advice of the Ethics Committee (CEUA/FCF/CAr: 09/2016) of the university. Cages were cleaned every 2 days.

\section{Device fabrication}

As previously described by our group, ${ }^{1}$ microfluidic chips composed of a single piece of polydimethylsiloxane (PDMS) obtained by the PSR technique without the bonding step and cleaning room facilities. As displayed in Figure S1 (A-C), 3D-printed cylinders in poly (lactic acid) with 2.0-mm diameter were used as support for the electrode channels, while the sample channel was defined by a stainless steel capillary with 700.0- $\mu$ m diameter, which was coated with AZ 50XT photoresist. We used a fused deposition modelling extrusion printer (3D Sethi3D AiPA3, Campinas, São Paulo, Brazil). The photoresist was deposited by centrifugation at $2000 \mathrm{rpm}$ for $10 \mathrm{~s}$ for three times and dried at room temperature during $20 \mathrm{~min}$. The scaffolds were supported on a 3D-printed pool-like piece (19 $\mathrm{mm} \times 59 \mathrm{~mm} \times 15 \mathrm{~mm}$ ). Photoresist prevented the filling of the scaffold joints between the sample and the electrode channels by polydimethylsiloxane (PDMS). Latter was prepared by mixing its monomer and curing agent at the ratio of 10:1 w/w. PDMS was cured at $60{ }^{\circ} \mathrm{C}$ in a laboratory oven (Blue $\mathrm{M}$, Blue Island, IL) for $1 \mathrm{~h}$ and, then hard baked for $30 \mathrm{~min}$ at $120^{\circ} \mathrm{C}$.

\section{Electrodes}

HB graphite cores were provided by Faber-Castell (São Carlos, Brazil). These cores show intermediate graphite-to-clay (G/C) ratios that define the core hardness in accordance with twenty $\mathrm{HB}$ scales, $9 \mathrm{H}$ up to $9 \mathrm{~B}$. As the core becomes softer through the increase in the quantity of graphite (higher $\mathrm{G} / \mathrm{C}$ ratios as indicated by the B scale; the letter ' $\mathrm{B}$ ' designates the pencil's mark blackness), it deposits more graphite leaving darker marks onto paper. In contrast, an increase in the amount of clay (lower $\mathrm{G} / \mathrm{C}$ ratios as indicated by the $\mathrm{H}$ scale; the letter ' $\mathrm{H}$ ' indicates the pencil's core hardness) creates harder cores that leaves lighter marks onto paper.'

Analyses by atomic force microscopy (AFM), scanning electronic microscopy (SEM), energy dispersive X-ray spectroscopy (EDS), and X-ray photoelectron spectroscopy (XPS) of the cores were made as described below. These cores were reversibly inserted above and below the sample channel as illustrated in Figure S1 (D), thus avoiding laborious routines to irreversibly attach the electrodes
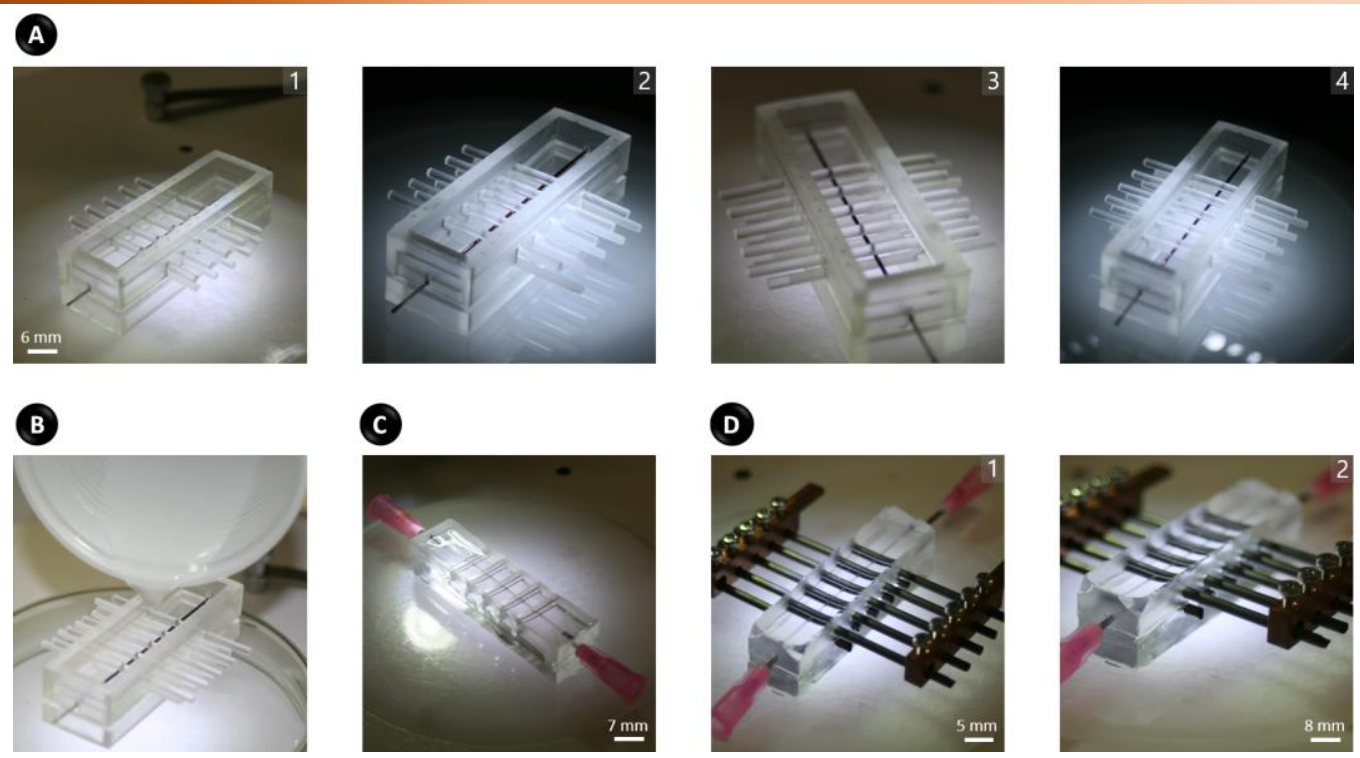

c

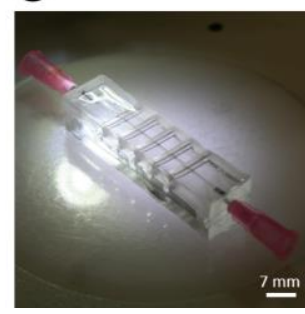

(D)

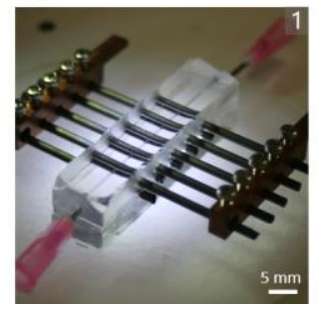

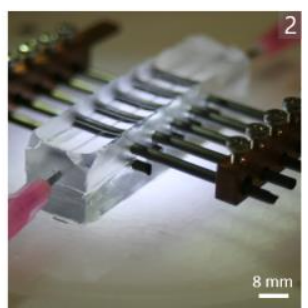

Figure S1. Fabrication, channels, and electrodes. (A) Scaffold (1-4), (B) addition of PDMS, (C) resulting PDMS device, and (D) chip with electrodes and copper pieces to achieved five parallel electric double-layer capacitors $(1,2)$. 
to the microfluidic device. ${ }^{2}$ The latter featured five pairs of pencil cores that were short-circuited with copper pieces to achieve an association of five polarizable capacitors in parallel.

AFM images (NX10 Park Systems, Santa Clara, CA) were obtained at $0.3 \mathrm{~Hz}$ in the tapping mode utilizing FMR Nanosensors tip (Neuchâtel, Switzerland). Electrical measurements by AFM for recording dC/dZ data were conducted using a Pt/Ir tip (NanoSensor PPP-EFM), nominal resonance of $75 \mathrm{kHz}$ and force constant of $2.8 \mathrm{~N} \mathrm{~m}^{-1}$. XPS analyses were performed in the Thermo Fisher Scientific K-Alpha spectrometer (Waltham, MA), while images of the electrodes studied were recorded by field emission SEM (FE-SEM, Zeiss Sigma, Jena, Germany) equipped with EDS operating at $3.0 \mathrm{kV}$.

\section{Impedance analyses}

The electrochemical assays were made in the portable PalmSens4 potentiostat (PalmSens, Houten, Netherlands) applying a sinewave voltage signal amplitude (peak-to-peak potential) of $25 \mathrm{mV}$ ac, integration time of $2 \mathrm{~s}$, frequencies from $10^{0}$ up to $10^{6} \mathrm{~Hz}$, and eventually dc voltages from -1.0 up to $+1.0 \mathrm{~V}$. The measurements were further conducted at stationary medium. In practice, plastic syringes were used to clean the chips with deionized water and change the samples. Differential capacitances $\left(C_{d}\right)$ were calculated from the data of impedance in agreement with the literature assuming the probes as pairs of polarizable electrodes. ${ }^{3-6}$ In this case, the equivalent electric circuit consists of a RC series circuit with solution resistance (Rsol) and $\mathrm{C}_{\mathrm{d}}$. While Rsol can be considered as the real part of impedance (Z'), $C_{d}$ can be calculated from the imaginary part of impedance (Z") and angular frequency $(\omega)$ as follows:

$\mathrm{Z}^{\prime \prime}=-\frac{1}{j \omega \mathrm{C}_{\mathrm{d}}}$

Then, substituting $\omega$ by $2 \pi f$ in which $f$ is the normal frequency, we have:

$\mathrm{C}_{\mathrm{d}}=-\frac{1}{j 2 \pi \mathrm{fZ}}$

\section{Extracellular vesicle biomarkers}

The sensor was applied to mice blood samples with and without Ehrlich tumor to make cancer diagnosis by analyzing extracellular vesicles (EVs) and their carried proteins. This tumor is a neoplasm that originates from female mice breast carcinoma. ${ }^{7}$ Ascites tumor was transplanted to the peritoneal region of mice and, then maintained by weekly replications of Ehrlich tumor cells, which present primary characteristics of mammary tumor cells and develop in a short term in mice. A volume of $200 \mu \mathrm{L}$ with $10^{7}$ cells per $\mathrm{mL}$ was injected intraperitoneally into 7 weeks old mice after local aseptic with iodinated alcohol.

For EVs isolation, blood samples were prepared through size exclusion chromatography (Izon qEV, Izon Science, Oxford, United Kingdom) using a fast ( $<10 \mathrm{~min}$ ) and low-cost $(<\$ 9.00$ per sample) protocol. The latter is further reported to generate intact EVs with reproducibility and samples pointedly purer when compared with ultracentrifugation method and precipitation reagent kits. ${ }^{\mathbf{8} 9}$ These techniques result in the co-isolation of contaminants such as protein aggregates. For the isolation of vesicles, $750 \mu \mathrm{L}$ of blood samples were loaded on columns pre-conditioned and eluted with PBS buffer. Three fractions of $500 \mu \mathrm{L}$ each were collected from each sample.

Images of isolate tumors were acquired by transmission electron microscopy (TEM) in a JEOL 1200 EXII (Peabody, MA) microscope, operated at $80 \mathrm{kV}$. Uranyl acetate (2\%) was added to the diluted samples and, then aliquots were dropped onto copper grids coated with a carbon film and dried at room temperature. The concentrations of EVs and carried proteins in 12 samples were obtained by nanoparticle tracking analysis (NTA) and Bradford protein assay, respectively. NTA analyses were accomplished on NanoSight NS300 (Malvern Instruments, Worcestershire, UK) equipped with $532 \mathrm{~nm}$ laser. The EVs were placed on sample chamber with sterile syringes until the liquid extended to the syringe tip. The measurements were performed in triplicate at room temperature. Protein cargos were quantified through the BioRad protein microassay approach (BioRad Laboratories $\mathrm{GmbH}$, Munchen, Germany). The concentrations of solubilized protein were achieved by recording absorbance responses at $595 \mathrm{~nm}$ (Beckman Coulter DU 800 spectrophotometer, Beckman Coulter, Brea, CA).

\section{Data treatment}

Unsupervised learning methods were used to assess dataset characteristics such as feature distribution and correlation. Clustering and dimensionality reduction tasks were performed by hierarchical clustering analysis (HCA) and principal component analysis (PCA), respectively. The feature data were first divided by the mean and scaled to unit variance to get a scale-independent dataset. These data were further treated by supervised machine learning methods for steps of classification and quantification. From algorithms available in the scikit-learn python library, ${ }^{10}$ pattern classifications were made by linear discriminant analysis (LDA), different classifiers such as random forests (RF) model, and sure independence screening and sparsifying operator (SISSO). Quantitative predictions were reached by regression using SISSO, partial least squares (PLS), and RF models. 


\section{Data chemical diversification by the core}

\subsection{Capacitance dispersion with dc potential}

Spectra of $C_{d} V s$. potential show chemical diversification owing to the different arrangement of ions on EDL for assuring interface electric balance. While cations are usually nonspecifically adsorbed at negative potentials because of their strong solvation, anions bearing a poorer hydration sheath can undergo specific adsorptions at positive ones. ${ }^{11,12}$ Nonspecific adsorptions comprise longrange electrostatic attractions (depend basically on the ion charge) and the charge balance is provided, e.g., by the excess of cations and deficiency of anions for negatively charged electrodes. Otherwise, specific adsorptions are based on short-range interactions (depend on the ion charge and nature) and lead to the excess of both the ions on EDL to ensure electroneutrality owing to the superequivalent adsorption phenomena. Nonspecific interactions face Brownian motion and are weaker than specific adsorptions, which typically lead to larger dispersions of $\mathrm{C}_{\mathrm{d}}$ with both dc potential and sine-wave frequency. ${ }^{\mathbf{5}}$

The spectra of $C_{d} v s$. potential to $\mathrm{KCl} 4.0 \mathrm{~mol} \mathrm{~L}^{-1}$ revealed different potentials of zero charge (PZC) to the electrode renewals as illustrated in Figure S2 (A,B), respectively. The electrical double-layer (EDL) capacitance governs the measured capacitance at these measurements with dc potential. A high electrolyte concentration was chosen to maximize the PZC-associated effects of the specific adsorptions on the Helmholtz layer because of the EDL narrowing in accordance with electrocapillarity equation and EDL GouyChapman model. ${ }^{11,12}$

All the PZCs were negative $(-0.31$ to $-0.38 \mathrm{~V})$, thus confirming the specific adsorption of anions on core graphite surfaces. These discrepancies disclose a heterogeneous chemical composition of the HB cores by revealing differences in the metal work function $\left(\Phi_{\mathrm{M}}\right)$ in accordance with the equation (3). ${ }^{12} \Phi_{\mathrm{M}}$ is the sum of electron chemical potential and electrode surface potential (formed by the quantum electronic spillover in agreement with jellium model), which reflect the composition and atomic structure of the material, respectively. $\mathrm{F}$ is the Faraday constant.

$\mathrm{PZC}=\frac{\Phi_{M}}{\mathrm{~F}}+$ constant

In addition, the differences in the PZCs further suggest heterogeneous surface areas of the HB cores since different roughness values of the electrodes favor the occurrence of specific adsorptions at EDL, ${ }^{\mathbf{5}}$ with consequent variation on PZC. PCA plots in Figure S2 (C) disclosed a clear separation of the data points, whereas the relative importance of the $\mathrm{C}_{\mathrm{d}}$ features for classification was attained from a generic RF model with tree depth of 25 and 500 estimators. Based on Figure S2 (D), a combination of $C_{d}$ values at negative and, specially, positive potentials around PZCs are the best to identify the samples. This greater importance of the positive potentials is likely due to the specific adsorption of anions as aforementioned. ${ }^{12}$

\subsection{Capacitance dispersion with frequency}

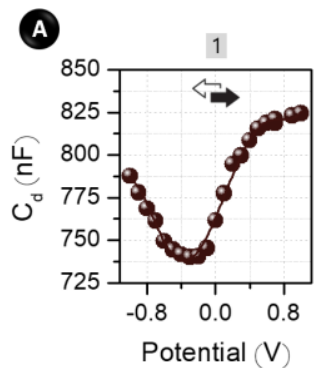

B

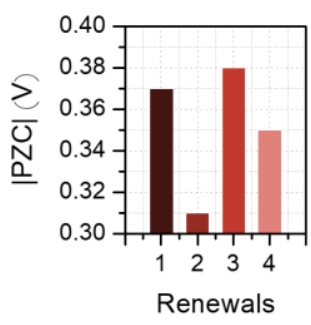

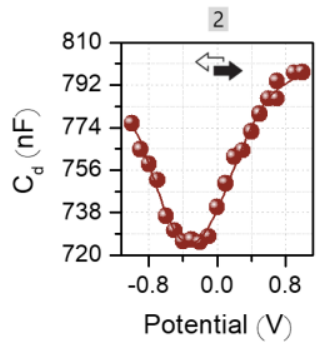

(C)

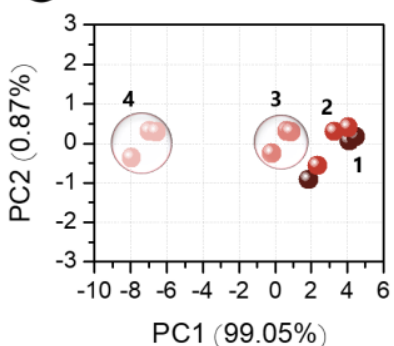

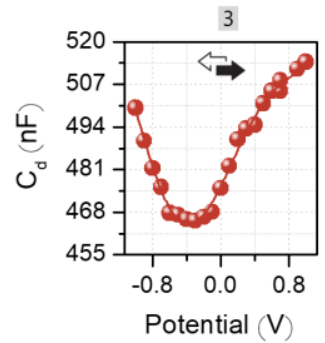
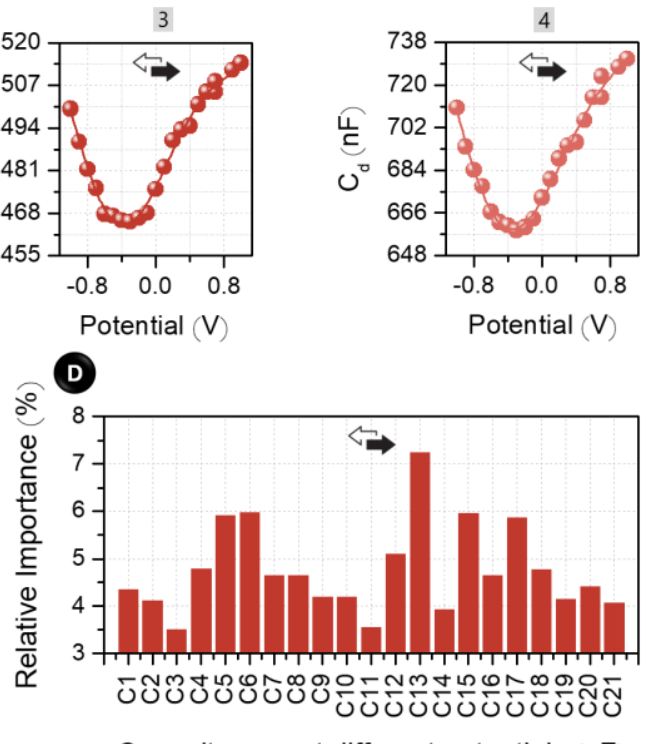

Capacitances at different potentials $(\mathrm{nF})$

Figure S2. Differential interactions by HB core. (A) Plots of average $\mathrm{C}_{\mathrm{d}}$ vs. potential for $\mathrm{KCl}$ solution at $4.0 \mathrm{~mol} \mathrm{~L}^{-1}$ recorded after renewing the cores (1-4). (B) PZC data (negative values in modulus) and (C) PCA scores plots related to the previous renewals. (D) Relative importance of the $C_{d}$ features for discriminatory capability of the RF algorithm. The black and white arrows in (A,D) indicate the features at positive and negative potentials. In (D), $\mathrm{C} 1-\mathrm{C} 21$ mean the capacitances ranging from -1.0 to $+1.0 \mathrm{~V}$, respectively, with changes of $0.1 \mathrm{~V}$. 
The spectrum of capacitance vs. frequency can be divided into two characteristic regions, low and high frequencies in which the EDL $\left(C_{d l}\right)$ and geometric capacitances $\left(C_{g}\right)$ govern $C_{d}$, respectively, as discussed in the main text. ${ }^{13,14} C_{d l}$ can be modelled by a series circuit encompassing metal $\left(\mathrm{C}_{\mathrm{M}}\right)$ and solution capacitances ( $\mathrm{CSsl}_{\mathrm{l}}$ ) as mathematically represented in the equation (4). $\mathrm{C}_{\mathrm{M}}$ is tightly tied to $\Phi_{M_{l}}{ }^{12}$ whereas $C_{s o l}$ is composed of series components in the Helmholtz $\left(C_{H}\right)$ and diffusive layers $\left(C_{D}\right)$. Regarding $C_{g}$, it is related to a capacitor formed by the electrodes (plates) with the sample acting as dielectric medium. Therefore, this parameter depends on the cell constant (gap and area of the plates) and Rsol.

$\frac{1}{C_{d}}=\frac{1}{C_{M}}+\frac{1}{C_{H}}+\frac{1}{C_{D}}$

Based on the previous discussion, the differences in the low-frequency $\mathrm{C}_{\mathrm{d}}$ features to $\mathrm{KCl} 4.0 \mathrm{~mol} \mathrm{~L}^{-1}$ in the four electrode renewals suggest heterogeneous chemical composition and area of the HB cores due to the relationships involving $\mathrm{C}_{\mathrm{dl}}$ with $\mathrm{C}_{\mathrm{M}}$ and $\mathrm{C}_{S \mathrm{~s}} \mathrm{l}_{\mathrm{l}}$ respectively. While the discrepancies in $\mathrm{C}_{\mathrm{M}}$ reveal distinct values of $\Phi_{\mathrm{M}}$, the variations in $\mathrm{Csol}_{\text {may }}$ be associated with distinct roughness of the electrodes by influencing the ion arrangement in the Helmholtz and diffusive layers. For instance, a high roughness favors the occurrence of specific adsorptions at EDL as previously described. ${ }^{5}$ Finally, since the analyses were performed to the same solution ( $\mathrm{KCl} 4.0 \mathrm{~mol} \mathrm{~L}^{-1}$; as a consequence, the bulk resistance remained constant) and the gap between the probes was constant over all the measurements, the differences in the high-frequency $C_{d}$ indicate heterogeneous areas of the probes.

The spectra of $\mathrm{C}_{d} v s$. frequency by the four renewed electrode pairs to $\mathrm{KCl} 4.0 \mathrm{~mol} \mathrm{~L}^{-1}$ was composed of 19 data features (C1 to C19) as shown in Figure 1 of main text. C1-C18 mean $C_{d}$ at the frequencies of 1.0, 2.2, and 4.6 on the order of $10^{\circ}, 10^{1}, 10^{2}, 10^{3}, 10^{4}$, and $10^{5} \mathrm{~Hz}$, respectively, whereas $\mathrm{C} 19$ means $\mathrm{C}_{\mathrm{d}}$ at $1.0 \times 10^{6} \mathrm{~Hz}$.

\section{Electrode characterization}

Capacitance gradient $(\mathrm{d} C / \mathrm{d} Z$ ) values by AFM electric analyses revealed only a small heterogeneity in 5 areas of $10 \mu \mathrm{m} \times 10 \mu \mathrm{m}$. The resulting data to 4 of these areas are shown in Figure $\mathbf{S 3}(\mathbf{A}-\mathbf{C})$. $d C / d Z$ differed by up to 0.06 a.u. only in a same area $(10 \mu \mathrm{m} \times$

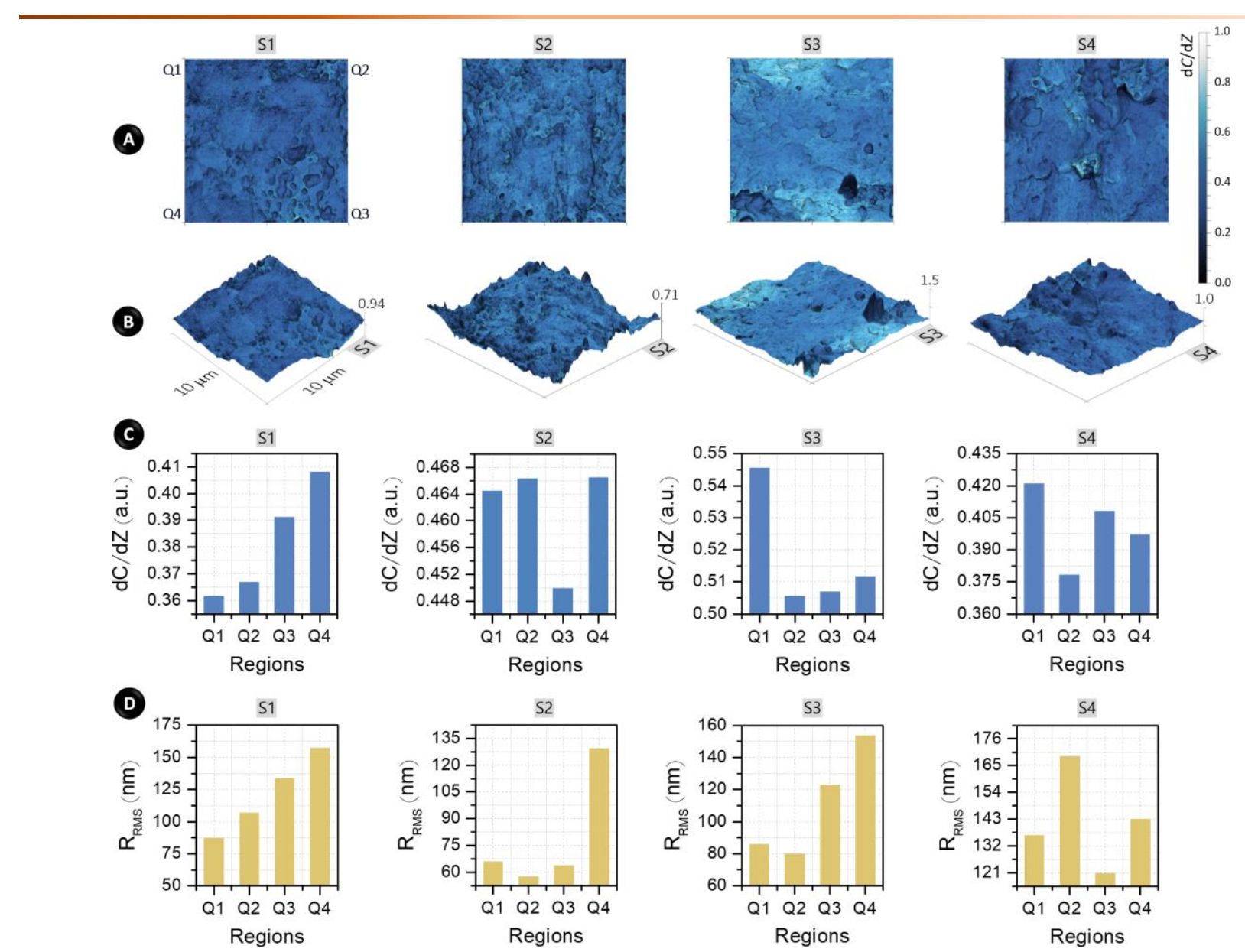

Figure S3. Heterogeneous topographical and electric properties of HB core. Data of (A) RRMs and (B) $d C / d Z$ of four different samples (S1-S4, 10 $\mu \mathrm{m}$ $\times 10 \mu \mathrm{m})$. (C) $\mathrm{d} C / \mathrm{d} Z$ data merged on 3D topographical $\mathrm{R}_{\mathrm{RMS}}$ images. The images in the same column are related to the same area. In (A,B), Q1-Q4 are quadrants of the analyzed regions. These definitions are also valid in (C). The samples S1,S2 are also shown in Figure $\mathbf{1}$ (F) of the main text. 
$10 \mu \mathrm{m})$. Its global value and standard deviation were 0.43 and 0.06 a.u., respectively. Otherwise, the root mean square roughnes ( $\mathrm{R}_{\mathrm{RMS}}$ ) values were heterogeneous in both the sceneries, within a same area of $10 \mu \mathrm{m} \times 10 \mu \mathrm{m}$ and globally with regard to five investigated regions, according to Figure S3 (D). RRMS differed by up to $75 \mathrm{~nm}$ in a same area, whereas its global value was $118.2 \mathrm{~nm}$ with standard deviation of $35.8 \mathrm{~nm}$.

2D SEM image in Figure S4 (A) reveals the core is composed of flake-graphite, the most usual natural graphitic material. Chemical composition of the cores was investigated by EDS and XPS. Chemical heterogeneity regarding the distribution of the pencil core constituents (graphite, clay, and wax) ${ }^{1}$ was first confirmed by monitoring the relative amounts of $\mathrm{C}, \mathrm{O}, \mathrm{Si}$, and $\mathrm{Al}$ in four regions (2.5 $\mu \mathrm{m} \times 2.5 \mu \mathrm{m})$ of a same area $(10 \mu \mathrm{m} \times 10 \mu \mathrm{m})$ by EDS. Whereas $\mathrm{C}$ is present in all of the three core constituents, $\mathrm{O}$ is found in clay and wax. Conversely, $\mathrm{Si}$ and $\mathrm{Al}$ are the major elements that compose the clay (e.g., minerals such as kaolinite: $\mathrm{Al}_{2} \mathrm{O}_{3} .2 \mathrm{SiO}_{2} \cdot \mathrm{H}_{2} \mathrm{O}$ ). $\mathrm{These}$ results are presented in Figure S4 (B). The global composition of the cores by EDS in Table $\mathbf{S 1}$ is in agreement with investigations addressed in the literature through X-ray fluorescence (XRF) analyses. ${ }^{15}$

We further checked the chemical heterogeneity in larger dimensions, namely, four areas of $500 \mu \mathrm{m} \times 500 \mu \mathrm{m}$ according to Figure S4 (C-E). The relative amounts of $\mathrm{C}, \mathrm{O}, \mathrm{Si}$, and $\mathrm{Al}$ demonstrated once again high discrepancy even when comparing these bigger regions. In special, the distributions of Si (approximately $9 \%$ to 15\%) and $\mathrm{Al}$ (about $7 \%$ to $15 \%$ ) revealed the clay on the core surface is highly heterogeneous. The amount profiles of $\mathrm{O}, \mathrm{Si}$, and $\mathrm{Al}$ were similar on each other in agreement with the occurrence of these species covalently bonded in kaolinite (clay). From this result, clay is the major source of $\mathrm{O}$. In addition to $\mathrm{C}=\mathrm{C}$ and $\mathrm{C}-\mathrm{C}$ peaks that are present in graphite, XPS results in Figure $\mathbf{S 4}(\mathbf{F})$ displayed the occurrence of $\mathrm{C}-\mathrm{O} / \mathrm{C}=\mathrm{O}$ peaks associated with fatty alcohols and
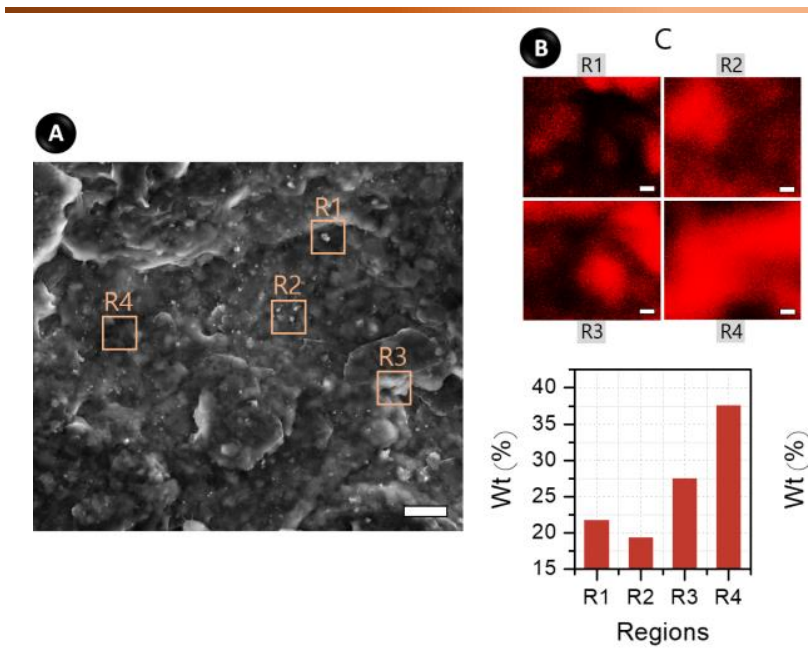
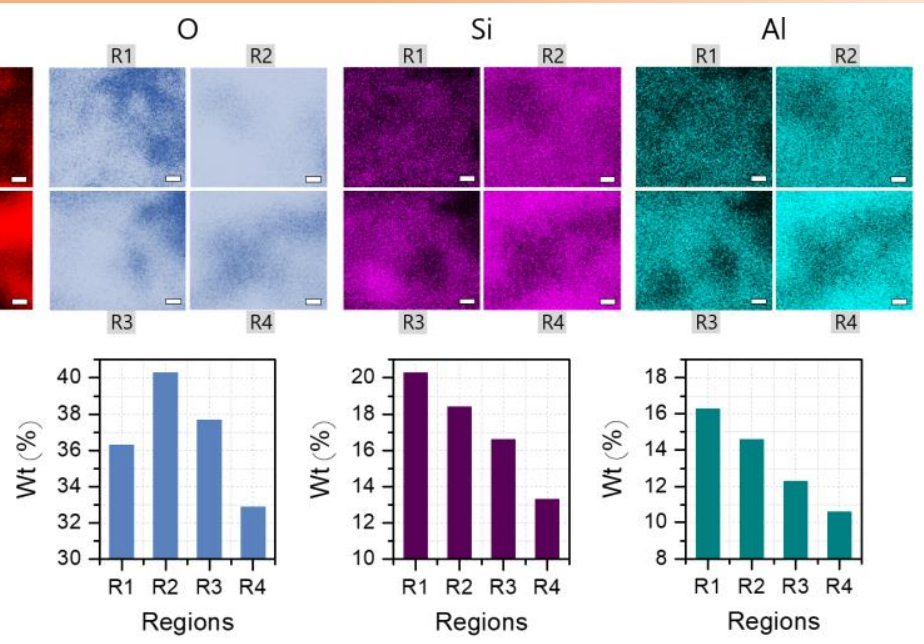
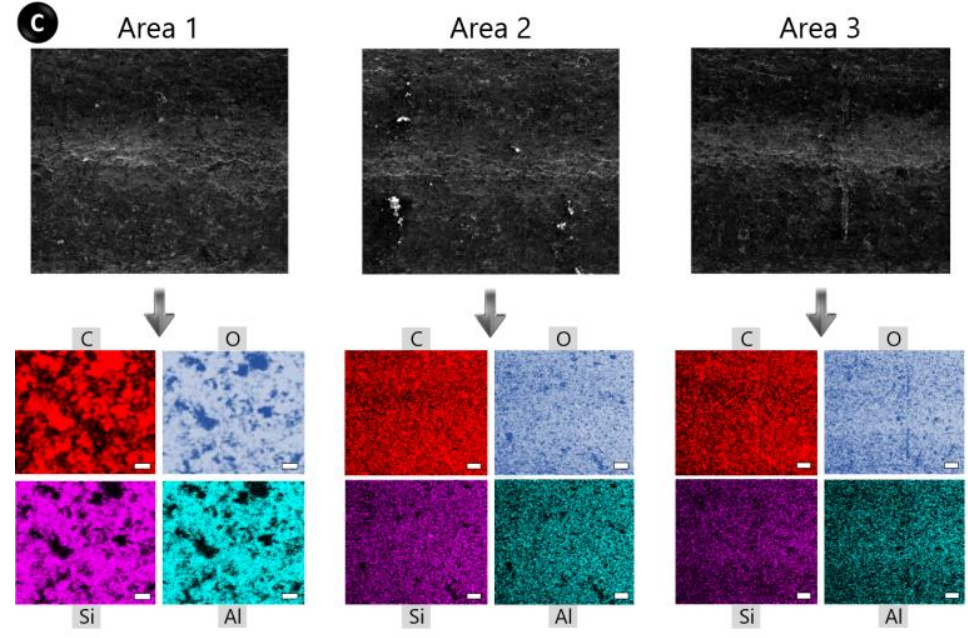

(D)
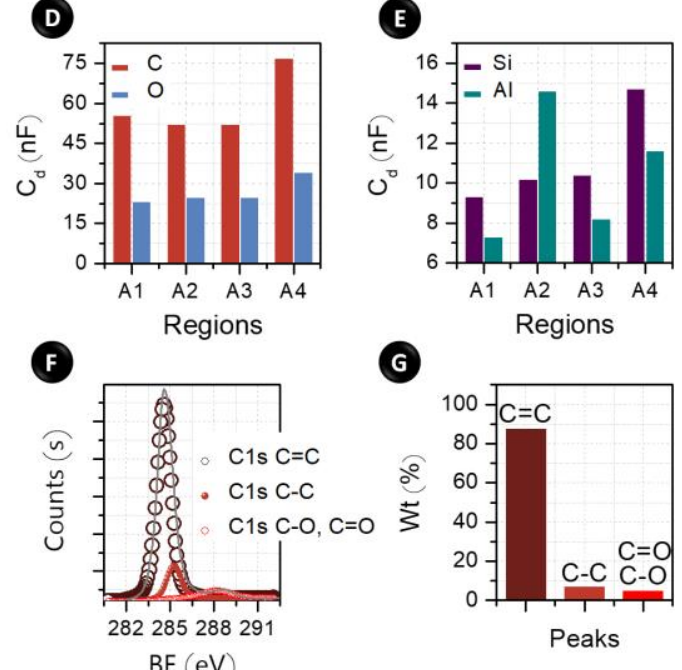

Figure S4. Heterogeneous chemical composition of HB core. (A) SEM image, (B) color maps on the amounts of C, O, Si, and Al by EDS in four distinct regions (R1-R4) of the first SEM image and resulting relative contents of these elements. (C) SEM images of distinct areas $(500 \mu \mathrm{m} \times 500 \mu \mathrm{m})$ and resulting color maps on the amounts of $\mathrm{C}, \mathrm{O}, \mathrm{Si}$, and $\mathrm{Al}$ as well as the relative contents of (D) C,O and (E) Si,Al. (F) High-resolution XPS spectrum of C1s peak and (G) relative amounts of the deconvoluted C peaks. In (A) and (B), the scale bars are $1 \mu \mathrm{m}$ and $250 \mathrm{~nm}$, respectively. In (C), the scale bars in maps associated with 'Area 1' are $25 \mu \mathrm{m}$, whereas the other bars correspond to $50 \mu \mathrm{m}$. 
Table S1. Overall relative composition (\%) of HB core by EDS

\begin{tabular}{lcccc} 
Elements & Region $\mathbf{1}$ & Region $\mathbf{2}$ & Region $\mathbf{3}$ & Region 4 \\
\hline C & 21.7 & 19.3 & 27.5 & 23.1 \\
Si & 20.3 & 18.4 & 16.6 & 9.3 \\
Al & 16.3 & 14.6 & 12.3 & 7.3 \\
$\mathbf{P}$ & 2.3 & 2.2 & 1.8 & 1.0 \\
Mg & 0.5 & 0.4 & 0.3 & - \\
\hline
\end{tabular}

acids in the wax, respectively. Such groups maximize intermolecular interactions with analytes, which are crucial for the discrimination ability of multidimensional sensors. ${ }^{16}$ The low amount of $\mathrm{C}-\mathrm{O}$ and $\mathrm{C}=\mathrm{O}$ groups as verified in Figure S4 (G) is in accordance with the lower quantity of wax on the cores. ${ }^{1}$

\section{Extracellular vesicle characterization}

Based on spectra of NTA in Figure S5, the tumor EVs (106 to $700 \mathrm{~nm}$ ) had a polydispersity greater than the healthy ones (156 to $348 \mathrm{~nm}$ ). Both these EVs were primarily composed of lipid structures with roughly $150 \mathrm{~nm}$ in diameter. Hence, the EVs mostly consist of exosomes (nanoscale vesicles, $\sim 50$ to $\sim 150 \mathrm{~nm}$ ) that are generated by the inward budding of the endosomal limiting membrane. ${ }^{17}$

\section{Classification of the mouse samples}

The sensor was capable of recognizing healthy and tumor sample groups by processing $15 \mathrm{C}_{\mathrm{d}}$ features through PCA as exhibited in the bode and scores plots $(n=8)$ of Figure $\mathbf{S 6}$ and $\mathbf{S 7}$, respectively. As for these features, $\mathrm{C} 1-\mathrm{C} 5 \mathrm{mean} \mathrm{C}_{\mathrm{d}}$ at $10.0,21.5,46.4 \mathrm{~Hz}$, 100.0, and $464.2 \mathrm{~Hz}$, respectively. C6-C14 correspond to $C_{d}$ at the frequencies of $10.0,21.5$, and 46.4 on the order of $10^{2}, 10^{3}$, and $10^{4}$ $\mathrm{Hz}$, respectively, whereas $\mathrm{C} 15$ corresponds to $\mathrm{C}_{\mathrm{d}}$ at $10.0 \times 10^{5} \mathrm{~Hz}$. The samples were randomly analyzed to avoid memory effect and reproducible $\mathrm{C}_{\mathrm{d}}$ data were attained to $\mathrm{KCl} 0.1 \mathrm{mmol} \mathrm{L}^{-1}$ before and after the EVs analysis, thus indicating the absence of contamination of the probes.
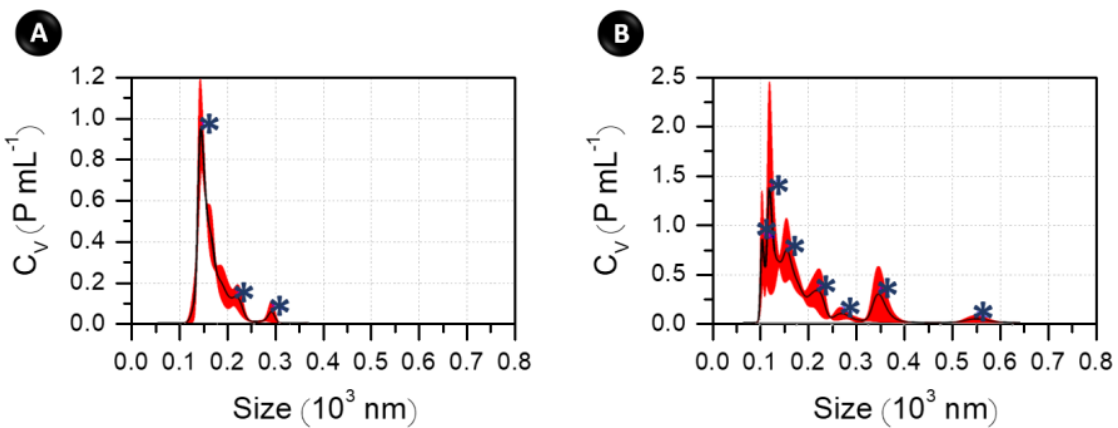

Figure S5. Analysis of EVs. NTA spectra of samples with isolated EVs originating from (A) healthy and (B) tumor mice.
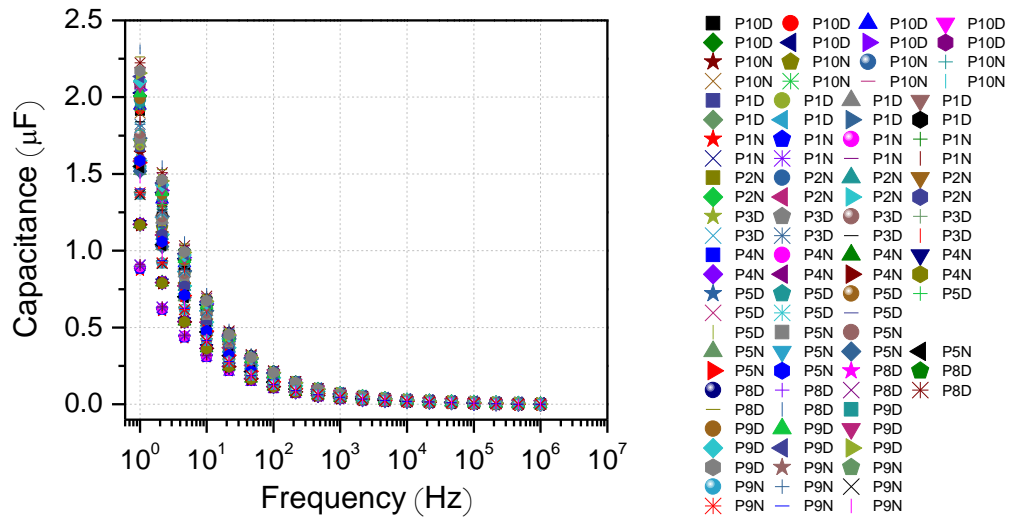

Figure S6. Analysis of EVs by the sensor. Plots of $\mathrm{C}_{\mathrm{d}}$ vs. frequency for the healthy and tumor treated mice samples. All the replicates $(n=8)$ are shown in the graphic. The identification of the samples in accordance with the definition in the main text is the following: 1 (P1N), 2 (P4N), 3 (P10N), 4 (P9N), 5 (P2N), 6 (P5N), 7 (P3D), 8 (P5D), 9 (P9D), 10 (P10D), 11 (P8D), and 12 (P1D). 


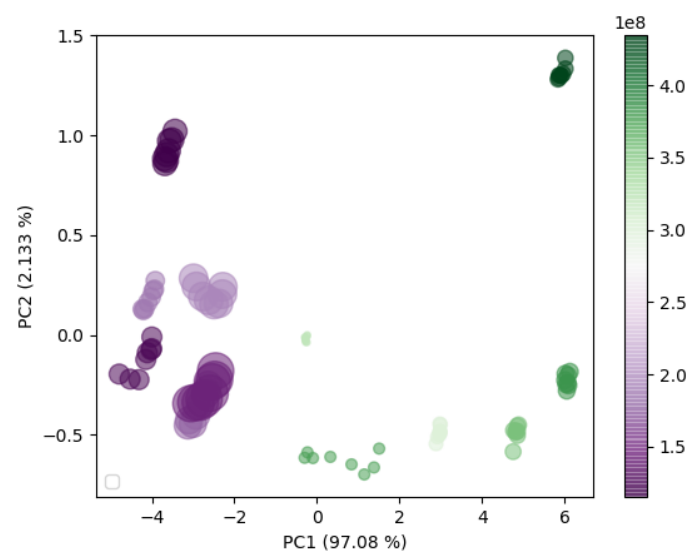

Figure S7. Analysis of EVs by the sensor. PCA plots for the recognition of the healthy (green) and tumor (purple) sample groups. All the replicates ( $n$ $=8$ ) are shown in the graphic. In this case, the size of the data points is directly associated with $C_{p}$ by Bradford assay, whereas the color scale presents relationship with the values of $C_{v}$ by NTA.

\section{Machine learning data to vesicle-containing mouse samples}

The constants $a$ and $b$ found for the optimized SISSO equations as shown in the main text for determination of the concentrations of EVs $\left(C_{V}\right)$ and carried proteins $\left(C_{p}\right)$ are equal to $-0.44 \times 10^{10}\left(a_{1}\right), 0.11 \times 10^{11}\left(a_{2}\right), 0.28 \times 10^{9}\left(b_{1}\right),-0.21 \times 10^{3}\left(a_{3}\right), 0.17 \times 10^{3}\left(a_{4}\right), a$ and $0.39 \times 10^{1}\left(b_{2}\right)$. An Android app was written with Xamarin (an open-source app platform) for calculating $C_{V}$ and $C_{P}$ on smartphone from SISSO. The values of these parameters and the resulting clinical status (healthy or diseased) are shown on smartphone display after each assay as shown in Video S1, which illustrates the analytical routine of this sample-to-answer fashion with two replicates. According to data by NTA and Bradford assay results, the thresholds for definition of the clinical status were $2.4 \times 10^{8} \mathrm{P} \mathrm{mL}{ }^{-1}$ and 2.2 $\mu \mathrm{g} \mathrm{L}^{-1}$. Concerning the multi-output regression using a single RF model (tree depth of 13 and 239 estimators), Figure S8 shows the absolute percentage errors associated with quantification of $C_{V}$ and $C_{p}$.

\section{Machine learning data to heavy metal-spiked lake samples}

The practical potential of the sensing device was also checked in the recognition of lake samples (from Campinas, Brazil) spiked with multiple heavy metal cations, i.e., $\mathrm{Ni}^{2+}, \mathrm{Fe}^{3+}$, and $\mathrm{Al}^{3}$ as shown in Table S2. A total of 30 samples was divided into 4 groups (A to D) in accordance with the safe limits by the World Health Organization (WHO) for $\mathrm{Cu}^{2+}(2.00){ }_{1}^{18} \mathrm{Ni}^{2+}(0.13),{ }^{19} \mathrm{and} \mathrm{Al}^{3+}\left(0.20 \mathrm{mg} \mathrm{L}^{-1}\right){ }^{20}$ The group A encompassed samples with total concentrations of metal cations below the global safe limit $\left(2.33 \mathrm{mg} \mathrm{L}^{-1}\right)$, whereas the other groups encompassed increasing concentrations above this limit.

Spectra of $C_{d} v s$. frequency were recorded $(n=5)$ by the sensor to the 30 samples of lake. Utilizing unsupervised and supervised methods of PCA and LDA, respectively, some samples of the groups $\mathbf{A}, \mathbf{B}$, and $\mathbf{C}$ were not clearly separated from each other as exhibited in plots of Figure $\mathbf{S 9}(\mathbf{A}, \mathbf{B})$, respectively. Accordingly, advanced supervised models were used with the intent to classify the groups and quantify the total and individual concentrations of the cations. Such tasks were based on randomized data splitting (ratios of $80 / 20$ and 75/25 for splitting the training/testing and training/validation samples, respectively) and 4-fold cross-validation.

RF model produced the largest accuracy ( 99\%) for the training/validation sets as depicted in Figure S9 (C). Further useful data provided by the RF classifier is the relative relevance of the features ( $C_{d}$ values at 10 frequencies as described in the figure caption)
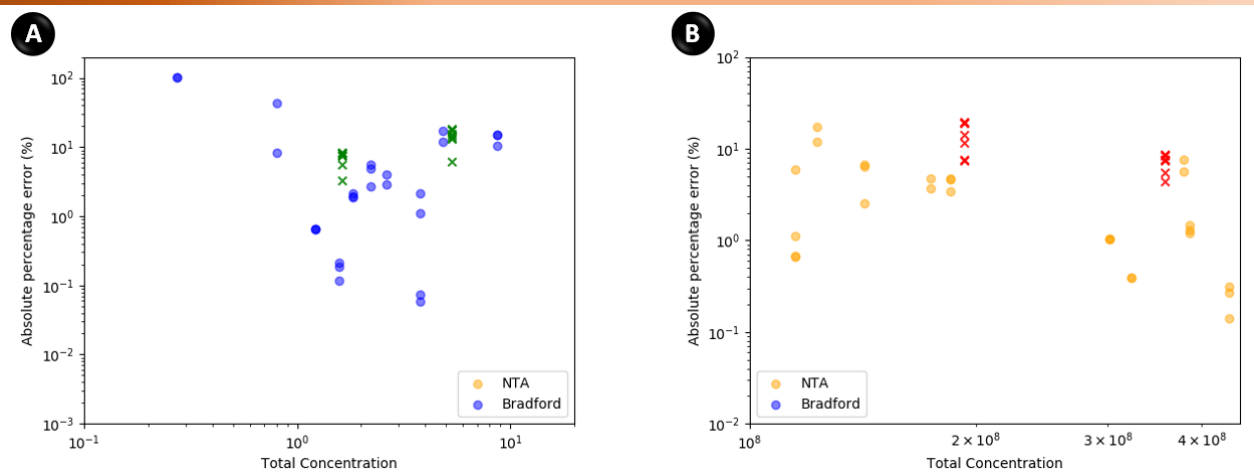

Figure S8. Analysis of EVs by the sensor. Absolute percentage errors for all the replicates $(n=8)$ related to the quantification of $(\mathbf{A}) C_{V}$ and $(\mathbf{B}) C_{P}$ by multi-output regression. Data points in circles and $\mathrm{x}$-format mean the validation and test subsets, respectively. 
Table S2. Lake samples spiked with multiple heavy metals

\begin{tabular}{|c|c|c|c|c|}
\hline \multirow[b]{2}{*}{ Total concentration $\left(\mathrm{mg} \mathrm{L}^{-1}\right)$} & \multirow[b]{2}{*}{ Sample } & \multicolumn{3}{|c|}{ Individual concentrations of the metal heavy cations $\left(\mathrm{mg} \mathrm{L}^{-1}\right)$} \\
\hline & & $\mathrm{Ni}^{2+}$ & $\mathrm{Al}^{3+}$ & $\mathrm{Cu}^{2+}$ \\
\hline 0.82 & A1 & 0.049 & 0.07 & 0.7 \\
\hline 0.94 & $\mathrm{~A} 2$ & 0.056 & 0.08 & 0.8 \\
\hline 1.05 & $\mathrm{~A} 3$ & 0.063 & 0.09 & 0.9 \\
\hline 1.17 & A4 & 0.07 & 0.1 & 1 \\
\hline 2.30 & B1 & 0.125 & 0.175 & 2 \\
\hline 2.53 & B2 & 0.1375 & 0.1925 & 2.2 \\
\hline 2.88 & B3 & 0.15625 & 0.21875 & 2.5 \\
\hline 3.11 & B4 & 0.16875 & 0.23625 & 2.7 \\
\hline 3.45 & B5 & 0.1875 & 0.2625 & 3 \\
\hline 5.75 & B7 & 0.3125 & 0.4375 & 5 \\
\hline 6.90 & B8 & 0.375 & 0.525 & 6 \\
\hline 8.05 & B9 & 0.4375 & 0.6125 & 7 \\
\hline 9.20 & B10 & 0.5 & 0.7 & 8 \\
\hline 11.70 & $\mathrm{C} 1$ & 0.7 & 1 & 10 \\
\hline 29.25 & $\mathrm{C} 2$ & 1.75 & 2.5 & 25 \\
\hline 58.50 & $\mathrm{C3}$ & 3.5 & 5 & 50 \\
\hline 70.20 & C4 & 4.2 & 6 & 60 \\
\hline 117.00 & C5 & 7 & 10 & 100 \\
\hline 234.00 & D1 & 14 & 20 & 200 \\
\hline 585.00 & D2 & 35 & 50 & 500 \\
\hline 760.50 & D3 & 45.5 & 65 & 650 \\
\hline 936.00 & D4 & 56 & 80 & 800 \\
\hline 1170.00 & D5 & 70 & 100 & 10000 \\
\hline
\end{tabular}

for classification according to Figure $\mathbf{S 9}$ (D). Preliminary assays confirmed a negligible importance of the values of $\mathrm{C}_{\mathrm{d}}$ governed by capacitor area $\left(>10^{4} \mathrm{~Hz}\right)$. Therefore, only the features associated with solution charging $\left(<10^{2} \mathrm{~Hz}\right)$ and electrode material $\left(10^{2}\right.$ to $10^{4}$ $\mathrm{Hz}$ ) were measured in these analyses. Both the capacitances contributed for recognizing the validation samples. In particular, a feature driven by the electrode material $\left(4.610^{2} \mathrm{~Hz}\right)$ exhibited the highest importance $(\sim 24 \%)$. Next, the RF method was successfully applied on the testing subset reaching $100.0 \%$ classification accuracy.

Accurate classification was also obtained from two pointedly simple equations encompassing only three $\mathrm{C}_{d}$ features as shown in Figure S9 (E). Such equations were reached by the SISSO model after cross-validation steps. Governed by solution charging, the selected $\mathrm{C}_{\mathrm{d}}$ provided $100.0 \%$ classification accuracy on both the validation and testing subsets. This decrease on data feature size is suitable for the development of sample-to-answer methods through the accomplishment of machine learning tasks on smartphone since the modelled equations can be easily executed in Android apps as discussed in the main text.

The low dispersion of the data points to each sample $(n=5)$ in the SISSO plots suggests a satisfactory precision of the chip. Our sensor bearing bare ready-to-use probes further exhibited a high lifetime and, then it is promising towards long-term applications. In practice, a different operator used the same device to fresh diluted solutions of the 30 spiked lake samples (Table S2). As shown in Figure S9 (F), the previously SISSO-modeled equations assured a pattern recognition accuracy of 100\% for these samples, which were assumed as testing subset in this case. This high lifetime is a key property for the daily applicability of the sensor by minimizing the negative effects of its poor reproducibility that arises from the heterogeneous surface of the graphite cores. These discrepancies led to a limited inter-probe precision.

Simultaneous quantification of the total and individual ions with accuracy was also provided by a multi-output regression using RF model with 11-tree depth and 347 estimators. The model optimization was once again conducted by means of an aleatory crossvalidation routine to the first 30 lake samples. The curves of predicted $v s$. expected concentration in Figure $\mathbf{S 9}(\mathbf{G})$ exhibited linear fittings close to ideal with correlation $\left(\mathrm{R}^{2}\right)>0.98$ and total mean absolute errors (MAEs) of $0.6\left(\mathrm{Ni}^{2+}\right), 0.8\left(\mathrm{Al}^{3+}\right), 8.0\left(\mathrm{Cu}^{2+}\right)$, and $9.4 \mathrm{mg}$ $\mathrm{L}^{-1}$ (total). These MAEs were most relevant for the lower ion amounts. The same RF model further led to accurate analyses of the total and individual ions in the second group of samples as shown in Figure $\mathbf{S 9} \mathbf{( H )}$ ), once again indicating high lifetime and reproducibility of the sensor. In this case, $\mathrm{R}^{2}$ was also $>0.98$ in all the cases and the errors were $0.5\left(\mathrm{Ni}^{2+}\right), 0.7\left(\mathrm{Al}^{3+}\right), 6.8\left(\mathrm{Cu}^{2+}\right)$, and $8.0 \mathrm{mg} \mathrm{L}^{-1}($ total).

\section{Specific determination of biomarker}



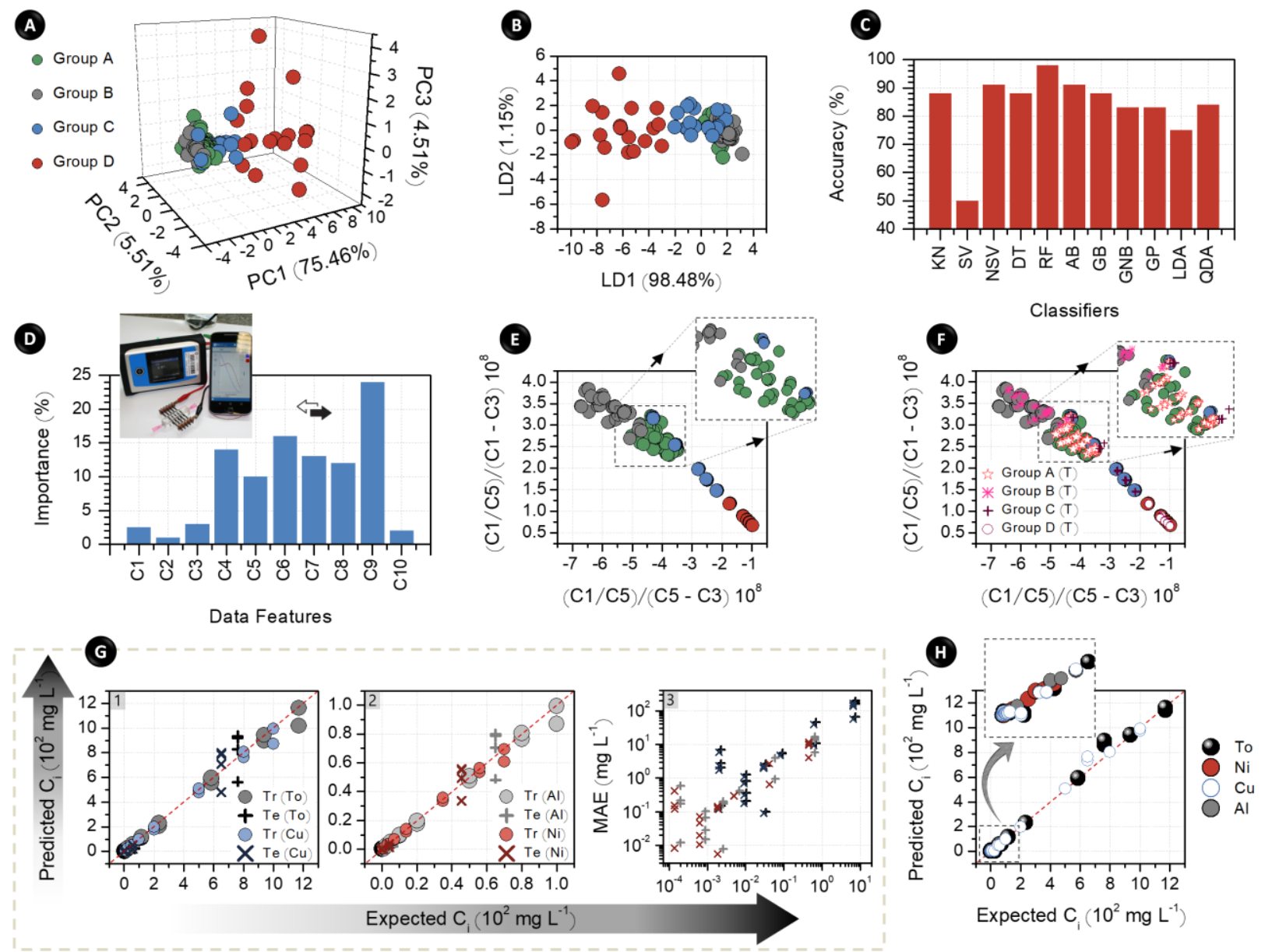

Figure S9. Machine learning results for lake mixings spiked with $\mathrm{Ni}^{2+}, \mathrm{Al}^{3+}$, and $\mathrm{Cu}^{2+}$. (A) PCA and (B) LDA plots. (C) Accuracy of various classifiers and (D) the resulting relative importance of $\mathrm{C}_{d}$ features for $\mathrm{RF}$ model-mediated classification of the validation samples. (E) Modeled SISSO map for the training subset and (F) sensor lifetime evaluation by applying this SISSO map to the second group of 30 lake samples (testing subset). (G) Fittings of predicted vs. expected concentrations of total (To) and individual metal cations for training (Tr) and testing (Te) subsets $(1,2)$ and resulting MAEs for the testing subset (3) from multi-output regression. (H) Sensor lifetime evaluation by applying the latter regression to the second group of 30 lake samples. The identification of the sample groups in (A) is true in (B,E,F). In (C), the abbreviations mean the classifiers, i.e., kneighbors (KN), support vector (SV), nu-SV (NSV), decision tree (DT), ada boosting (AB), gradient boosting (GB), gaussian nb (GNB), gaussian process (GP), and quadratic discriminant analysis (QDA) beyond the already defined LDA and RF models. In (D), C1-C9 mean $C_{d}$ at the frequencies of 1.0, 2.2, and 4.6 on the order of $10^{0}, 10^{1}$, and $10^{2} \mathrm{~Hz}$, respectively, whereas $\mathrm{C} 10$ corresponds to $C_{d}$ at $1.0 \times 10^{3} \mathrm{~Hz}$. These definitions are also true in (E,F). The inset in (D) shows a photo of the setup with portable potentiostat, device, and smartphone. The black and white arrows indicate the data of $\mathrm{C}_{\mathrm{d}}$ at frequencies lower and higher than $10^{2} \mathrm{~Hz}$. The insets in $(\mathbf{E}, \mathbf{F}, \mathbf{H})$ show amplified views of the highlighted regions. In $(\mathbf{F})$, the testing subsets are highlighted with ' $(T)^{\prime}$. In $(\mathbf{G})$, the definitions in $(1,2)$ are also valid for $(3)$. In $(\mathbf{G}, \mathbf{H})$, the red dashed lines mean the ideal behavior (unitary slope and intercept on 0 ).

Preliminary assays were also conducted to demonstrate the utility of the sensor for specific quantification. Briefly, the sensor was applied to determine peptide p16, a prostate cancer biomarker, after its immunocapture by antibody-anchored magnetic beads (MB$\mathrm{Ab}$ ). After an incubation time, the formed conjugates MB-Ab-p16 were isolated with the aid of an external magnet and resuspended in water. Such steps aimed to eliminate interferents and, then to provide accurate recognition assays by the sensor. The peptide $\mathrm{p} 16$ presents 4 ankyrin repeats and its expression is tightly tied to the incidence and stage of prostate and cervical tumors. ${ }^{21}$ In this regard, this specie is used as a biomarker towards the diagnosis and prognosis of these tumors. p16 can be further monitored towards therapeutic evaluation since its expression decreases with chemotherapy and radiotherapy procedures. ${ }^{22}$

Antibodies (Ab) were conjugated to MBs as previously reported..$^{23}$ For this purpose, $200 \mu \mathrm{L}$ of MB were transferred to a microtube containing $1 \mathrm{~mL}$ of MES buffer (15 mmol L-1, pH 6.0), and vortexed for $30 \mathrm{~s}$. Then, the MBs were magnetically separated for $2 \mathrm{~min}$ and the supernatant was removed (this step was repeated 3 times). Subsequently, these MBs were resuspended in $1 \mathrm{~mL}$ of EDC (10 $\mathrm{mg} \mathrm{mL}^{-1}$ in MES buffer) and incubated under slow stirring for $30 \mathrm{~min}$ at room temperature. After this time, the MBs were magnetically separated and $1 \mathrm{~mL}$ of MES containing the $A b\left(10 \mu \mathrm{g} \mathrm{mL}^{-1}\right)$ was added and incubated with slow stirring overnight. After this time, the MBs-Ab were washed with $1 \mathrm{~mL}$ of MES three times and dispersed in $400 \mu \mathrm{L}$ of PBS-T20 with $0.1 \% \mathrm{v} / \mathrm{v}$ to BSA for blocking nonbioconjugated active sites on MB surfaces. 
More specifically, our sensor was used in the quantification of p16 standards (diluted in PBS solution at concentrations ranging from $5.0 \mathrm{pg} \mathrm{mL}^{-1}$ to $0.1 \mathrm{\mu g} \mathrm{mL}^{-1}$ ) after immunoassays as depicted in Figure 3 (B) of the main text. This routine was based on magnetic beads chemically modified with $\mathrm{p} 16$ antibody, MBs-Ab, for $\mathrm{p} 16$ capture. MB-Ab dispersion (5.6\% v/v MBs-Ab to PBS buffer) was first added to the sample for 16 capture under stirring for $30 \mathrm{~min}$. In this step, $20 \mathrm{uL}$ of MB-Ab were added to $340 \mathrm{uL}$ of buffer (PBS, pH 7.4) containing $\mathrm{p} 16$ at the desired concentration. After, this target was insulated from sample by applying an external magnetic field with the aid of a magnet. Next, the formed conjugates MB-Ab-p16 were washed with $400 \mu \mathrm{L}$ of deionized water ( 3 times) and enriched by resuspension in $125 \mu \mathrm{L}$ of water as well. Finally, the resulting medium was inserted into the device for impedance measurements. Importantly, for cleaning the PDMS device between the measurements, we used pumped $1 \mathrm{~mL}$ of ethanol and then deionized water with the aid of plastic syringes.

The sensor achieved a limit-of-detection (LOD) of $0.6 \mathrm{pg} \mathrm{mL}^{-1}$ that is lower than the data reported in the literature. A piezoelectric immunosensor was developed by Yang et a/.21 for the detection of p16 using a quartz microbalance. P16 polyclonal antibody was attached on piezoelectric crystal surface in an orderly orientation using protein A. The device showed a LOD of $10 \mathrm{ng} \mathrm{mL} \mathrm{L}^{-1}$. Duangkaew et al. ${ }^{22}$ described a sensitive electrochemical immunosensor for specific detection of p16 employing sandwich assembly with screenprinted electrodes and double amplification (gold nanoparticles conjugated on secondary antibody and sequential deposition of gold and silver layers). This method provided a LOD of $1.3 \mathrm{ng} \mathrm{mL}^{-1}$ and the quantification of $\mathrm{p} 16$ in cell lysates obtained from patients. Wang et al. ${ }^{24}$ described an electrogenerated chemiluminescence biosensor for the detection of anti-oncogene CdkN2A/p16 from

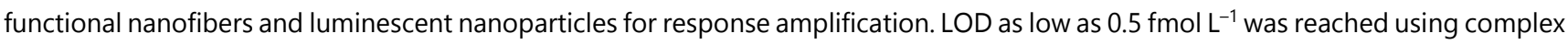
conjugates, composed of polycaprolactam 6 electrospun nanofibers, carboxylated multiwalled carbon nanotubes, $\mathrm{SiO}_{2}$ nanoparticles, and single-stranded DNA (ssDNA).

Magnetic immunoassays have provided the accurate determination of circulating cancer biomarkers, ${ }^{2,23}$ including EV-associated cargos. ${ }^{25-37}$ Herein, our sensor could directly detect the target in a sensitive and simple fashion, dispensing the use of either primary antibodies covalently attached on electrode surface or active probes on secondary antibodies as commonly needed. Remarkably, the sensibility of our sensor can be further increased by simply increasing the number of pencil graphite cores acting as parallel capacitors of the device. ${ }^{2}$ Our sensor further presented low consumption of samples. While analyses to real samples are necessary to evaluate its effective potential, such results suggest the sensor may be also attractive for clinical diagnoses by quantifying single targets in a specific fashion.

\section{Video caption}

Video S1 illustrates the analysis process using smartphone to control a portable potentiostat, acquire the capacitance data, and make the machine learning tasks. The concentrations of vesicles and proteins, along with the clinical status (healthy or diseased), are exhibited on smartphone display after the assay.

\section{References}

1. Nicoliche, C. Y. N.; Costa, G. F.; Gobbi, A. L.; Shimizu, F. M.; Lima, R. S. Pencil graphite core for pattern recognition applications. Chem. Commun. 2019, 55, 4623-4626.

2. de Oliveira, R. A. G.; Nicoliche, C. Y. N.; Pasqualeti, A. M.; Shimizu, F. M.; Ribeiro, I. R.; Melendez, M. E.; Carvalho, A. L.; Gobbi, A. L.; Faria, R. C.; Lima, R. S. Low-Cost and Rapid-Production Microfluidic Electrochemical Double-Layer Capacitors for Fast and Sensitive Breast Cancer Diagnosis. Anal. Chem. 2018, 90, 12377-12384.

3. Itagaki, M.; Suzuki, S.; Shitanda, I.; Watanabe, K. Electrochemical Impedance and Complex Capacitance to Interpret Electrochemical Capacitor. Electrochemistry 2007, 75, 649-655.

4. Chinaglia, D. L.; Gozzi, G.; Alfaro, R. A. M.; Hessel, R. Espectroscopia de impedância no laboratório de ensino. Rev. Bras. Ens. Fis. $2008,30,4504$.

5. Lockett, V.; Sedev, R.; Ralston, J.; Horne, M.; Rodopoulos, T. Differential Capacitance of the Electrical Double Layer in Imidazolium-Based lonic Liquids: Influence of Potential, Cation Size, and Temperature. J. Phys. Chem. C 2008, 112, 7486-7495.

6. Ji, H.; Zhao, X.; Qiao, Z.; Jung, J.; Zhu, Y.; Lu, Y.; Zang, L. L.; MacDonald, A. H.; Ruoff, R. S. Capacitance of Carbon-Based Electrical Double-Layer Capacitors, Nat. Commun. 2014, 5, 3317.

7. Mishra, S.; Tamta, A. K.; Sarikhani, M.; Desingu, P. A.; Kizkekra, S. M.; Pandit, A. S.; Kumar, S.; Khan, D.; Raghavan, S. C.; Sundaresan, N. R. Subcutaneous Ehrlich Ascites Carcinoma mice model for studying cancer-induced cardiomyopathy. Sci. Rep. 2018, 8, 5599.

8. Böing, A. N.; Pol, E. V. D.; Grootemaat, A. E.; Coumans, F. A. W.; Sturk, A.; Nieuwland, R. Single-step isolation of extracellular vesicles by sizeexclusion chromatography. J. Extracell. Vesicles 2014, 3, 23430.

9. Lobb, R. J.; Becker, M.; Wen, S. W.; Wong, C. S.; Wiegmans, A. P.; Leimgruber, A.; Möller, A. Optimized exosome isolation protocol for cell culture supernatant and human plasma. J. Extracell. Vesicles 2015, 4, 27031.

10. Pedregosa, F.; Varoquaux, G.; Gramfort, A.; Michel, V.; Thirion, B.; Grisel, O.; Blondel, M.; Prettenhofer, P.; Weiss, R.; Dubourg, V.; Vanderplas, J.; Passos, A.; Cournapeau, D.; Brucher, M.; Perrot, M.; Duchesnay, E. Scikit-learn: Machine Learning in Python. J. Mach. Learn. Res. 2011, 12, 28252830.

11. Bard, A. L.; Faulkner, L. R. Electrochemical Methods: Fundamentals and Applications, 2nd ed., John Wiley \& Sons, New York, 2001, pp. 368-534. 
12. Bockris, J. O'M.; Reddy, A. K. N.; Gamboa-Aldeco, M. Modern Electrochemistry: Fundamentals of Electrodics $2 \mathrm{~A}$, Vol. 2, Kluwer Academic Publishers, New York, 2002, pp. 806-919.

13. Oliveira, R. F.; Merces, L.; Vello, T. P.; Bufon, C. B. F. Water-gated phthalocyanine transistors: Operation and transduction of the peptideeenzyme interaction. Org. Electron. 2016, 31, 217-226.

14. Riul Jr., A.; Malmegrim, R. R.; Fonseca, F. J.; Mattoso, L. H. C. An artificial taste sensor based on conducting polymers. Biosens. Bioelectron. 2003, 18, 1365-1369.

15. Bhowmik, R. N. Ferromagnetism in lead graphite-pencils and magnetic composite with $\mathrm{CoFe}_{2} \mathrm{O}_{4}$ particles. Compos. Part B-Eng. 2012, 43, 503509.

16. Tao, Y.; Ran, X.; Ren, J.; Qu, X. Array-Based Sensing of Proteins and Bacteria By Using Multiple Luminescent Nanodots as Fluorescent Probes. Smal/ 2014, 10, 3667-3671.

17. Lim, C. Z. J.; Zhang, L.; Zhang, Y.; Sundah, N. R.; Shao, H. New Sensors for Extracellular Vesicles: Insights on Constituent and Associated Biomarkers. ACS Sens. 2020, 5, 4-12.

18. WHO, World Health Organization, Copper in drinking-water: Background document for development of WHO Guidelines for Drinking-water Quality 2004.

19. WHO, World Health Organization, Nickel in drinking-water: Background document for development of WHO Guidelines for Drinking-water Quality 2005.

20. WHO, World Health Organization, Aluminum in drinking-water: Background document for development of WHO Guidelines for Drinking-water Quality 2010.

21. Yang, L.; Huang, X.; Sun, L.; Xu, L. A piezoelectric immunosensor for the rapid detection of p16 ${ }^{\text {INK4a }}$ expression in liquid-based cervical cytology specimens. Sens. Actuat. B 2016, 224, 863-867.

22. Duangkaew, P.; Tapaneeyakorn, S.; Apiwat, C.; Dharakul, T.; Laiwejpithay, S.; Kanatharana, P.; Laocharoensuk, R. Ultrasensitive electrochemical immunosensor based on dual signal amplification process for $\mathrm{p} 16^{\mathrm{INK} 4 \mathrm{a}}$ cervical cancer detection in clinical samples. Biosens. Bioelectron. 2015, $74,673-679$.

23. de Oliveira, R. A. G.; Materon, E. M.; Melendez, M. E.; Carvalho, A. L.; Faria, R. C. Disposable Microfluidic Immunoarray Device for Sensitive Breast Cancer Biomarker Detection. ACS Appl. Mater. Interfaces 2017, 9, 27433-27440.

24. Wang, X.; Wang, Y.; Shan, Y.; Jiang, M.; Gong, M. Jin, X.; Wang, X.; Cheng, J. An electrochemiluminescence biosensor for detection of CdkN2A/p16 anti-oncogene based on functional electrospun nanofibers and core-shell luminescent composite nanoparticles. Talanta 2018, $187,179-187$.

25. Shao, H.; Chung, J.; Lee, K.; Balaj, L.; Min, C.; Carter, B. S.; Hochberg, F. H.; Breakefield, X. O.; Lee, H.; Weissleder, R. Chip-based analysis of exosomal mRNA mediating drug resistance in glioblastoma. Nat. Commun. 2015, 6, 6999.

26. Jeong, S.; Park, J.; Pathania, D.; Castro, C. M.; Weissleder, R.; Lee, H. Integrated Magneto-Electrochemical Sensor for Exosome Analysis. ACS Nano. 2016, 10, 1802-1809.

27. Zhao, Z.; Yang, Y.; Zeng, Y.; He, M. A. A microfluidic ExoSearch chip for multiplexed exosome detection towards blood-based ovarian cancer diagnosis. Lab Chip 2016, 16, 489-496.

28. Zong, S.; Wang, L.; Chen, C.; Lu, J.; Zhu, D.; Zhang, Y.; Wang, Z.; Cui, Y. Facile detection of tumor-derived exosomes using magnetic nanobeads and SERS nanoprobes. Anal. Methods 2016, 8, 5001-5008.

29. Liang, K.; Liu, F.; Fan, J.; Sun, D.; Liu, C.; Lyon, C. J.; Bernard, D. W.; Li, Y.; Yokoi, K.; Katz, M. H. Nanoplasmonic Quantification of Tumor-derived Extracellular Vesicles in Plasma Microsamples for Diagnosis and Treatment Monitoring. Nat. Biomed. Eng. 2017, 1, 0021.

30. Jiang, Y.; Shi, M.; Liu, Y.; Wan, S.; Cui, C.; Zhang, L.; Tan, W. Aptamer/AuNP Biosensor for Colorimetric Profiling of Exosomal Proteins. Angew. Chem. Int. Ed. Engl. 2017, 56, 11916-11920.

31. Ko, J.; Bhagwat, N.; Yee, S. S.; Ortiz, N.; Sahmoud, A.; Black, T.; Aiello, N. M.; McKenzie, L.; O’Hara, M.; Redlinger, C.; Romeo, J.; Carpenter, E. L.; Stanger, B. Z.; Issadore, D. Combining Machine Learning and Nanofluidic Technology To Diagnose Pancreatic Cancer Using Exosomes. ACS Nano. 2017, 11, 11182-11193.

32. Tavallaie, R.; McCarroll, J.; Le Grand, M.; Ariotti, N.; Schuhmann, W.; Bakker, E.; Tilley, R. D.; Hibbert, D. B.; Kavallaris, M.; Gooding, J. J. Nucleic acid hybridization on an electrically reconfigurable network of gold-coated magnetic nanoparticles enables microRNA detection in blood. Nat. Nanotechnol. 2018, 13, 1066-1071.

33. Lee, K.; Fraser, K.; Ghaddar, B.; Yang, K.; Kim, E.; Balaj, L.; Chiocca, E. A.; Breakefield, X. O.; Lee, H.; Weissleder, R. Multiplexed Profiling of Single Extracellular Vesicles. ACS Nano. 2018, 12, 494-503.

34. Liu, C.; Xu, X.; Li, B.; Situ, B.; Pan, W.; Hu, Y.; An, T.; Yao, S.; Zheng, L. Single-Exosome-Counting Immunoassays for Cancer Diagnostics. Nano Lett. 2018, 18, 4226-4232.

35. Lim, C. Z. J.; Zhang, Y.; Chen, Y.; Zhao, H.; Stephenson, M. C.; Ho, N. R. Y.; Chen, Y.; Chung, J. Reilhac, A.; Loh, T. P.; Chen, C. L. H.; Shao, H. Subtyping of circulating exosome-bound amyloid $\beta$ reflects brain plaque deposition. Nat. Commun. 2019, $10,1144$.

36. Wang, Z.; Sun, X. Natalia, A.; Tang, C. S. L.; Ang, C. B. T.; Ong, C. A. J.; Teo, M. C. C.; So, J. B. Y.; Shao, H. Dual-Selective Magnetic Analysis of Extracellular Vesicle Glycans. Matter 2019, 2, 150-166.

37. Mathew, D. G.; Beekman, P.; Lemay, S. G.; Zuilhof, H.; Le Gac, S.; van der Wiel, W. G. Electrochemical Detection of Tumor-Derived Extracellular Vesicles on Nanointerdigitated Electrodes. Nano Lett. 2020, 20, 820-828. 